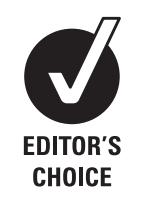

See Commentary, p 331

- Additional materials are published online only. To view these files please visit the journal online (http://gut.bmj. com/content/61/3.toc).

${ }^{1}$ Swiss Institute of Allergy and Asthma Research (SIAF), University of Zurich, Zurich, Switzerland

${ }^{2}$ Alimentary Health Ltd., Cork, Ireland

${ }^{3}$ Alimentary Pharmabiotic Centre, University College Cork, Cork, Ireland

\section{Correspondence to} Dr Liam O'Mahony, SIAF, Obere Strasse 22, Davos Platz 7270, Zurich Switzerland; liam.omahony@siaf.uzh.ch

Authors PK and DG contributed equally and share first authorship.

Revised 8 September 2011 Accepted 25 September 2011 Published Online First

3 November 2011

\title{
Bifidobacterium infantis 35624 administration induces Foxp3 T regulatory cells in human peripheral blood: potential role for myeloid and plasmacytoid dendritic cells
}

\author{
Patrycja Konieczna, ${ }_{1}^{1}$ David Groeger, ${ }^{2}$ Mario Ziegler, ${ }^{1}$ Remo Frei, ${ }^{1}$ Ruth Ferstl, ${ }^{1}$ \\ Fergus Shanahan, ${ }^{3}$ Eamonn M M Quigley, ${ }^{3}$ Barry Kiely, ${ }^{2}$ Cezmi A Akdis, \\ Liam O'Mahony ${ }^{1}$
}

\section{ABSTRACT}

Background Intestinal homoeostasis is dependent on immunological tolerance to the microbiota.

Objective To (1) determine if a probiotic could induce Foxp3 T cells in humans; (2) to elucidate the molecular mechanisms, which are involved in the induction of Foxp3 T cells by human dendritic cells.

Design Cytokine secretion and Foxp3 expression were assessed in human volunteers following Bifidobacterium infantis feeding. Monocyte-derived dendritic cells (MDDCs), myeloid dendritic cells (mDCs) and plasmacytoid dendritic cells ( $\mathrm{pDCs}$ ) were incubated in vitro with $B$ infantis and autologous lymphocytes. Transcription factor expression, costimulatory molecule expression, cytokine secretion, retinoic acid and tryptophan metabolism were analysed.

Results Volunteers fed $B$ infantis displayed a selective increase in secretion of interleukin (IL)-10 and enhanced Foxp3 expression in peripheral blood. In vitro, MDDCs, $\mathrm{mDCs}$ and $\mathrm{pDCs}$ expressed indoleamine 2,3-dioxygenase and secreted $\mathrm{IL}-10$, but not $\mathrm{IL}-12 \mathrm{p} 70$, in response to $B$ infantis. MDDC and $\mathrm{mDC}$ IL-10 secretion was Toll-like receptor (TLR)-2/6 dependent, while pDC IL-10 secretion was TLR-9 dependent. In addition, MDDCs and mDCs expressed RALDH2, which was TLR-2 and DC-SIGN dependent. $B$ infantis-stimulated MDDCs, mDCs and pDCs induced T cell Foxp3 expression. TLR-2, DC-SIGN and retinoic acid were required for $\mathrm{MDDC}$ and $\mathrm{mDC}$ induction of Foxp3 T cells, while pDCs required indoleamine 2,3-dioxygenase.

Conclusions $B$ infantis administration to humans selectively promotes immunoregulatory responses, suggesting that this microbe may have therapeutic utility in patients with inflammatory disease. Cross-talk between multiple pattern-recognition receptors and metabolic pathways determines the innate and subsequent $T$ regulatory cell response to $B$ infantis. These findings link nutrition, microbiota and the induction of tolerance within the gastrointestinal mucosa.

\section{INTRODUCTION}

The commensal microbiota is required for optimal host development and for ongoing intestinal homoeostasis, which involves an interdependence between microbes and immunity. ${ }^{1-3}$ This requires discriminative responses to commensals in

\section{Significance of this study}

What is already known about this subject?

- Commensal microbiota are important for intestinal homoeostasis.

- $\mathrm{T}$ regulatory cells are required for intestinal tolerance.

- Dendritic cells induce T regulatory cells within the intestine.

- Murine models show that microbiota-dendritic cell interactions induce $\mathrm{T}$ regulatory cells.

What are the new findings?

- Bifidobacterium infantis feeding induces Foxp3 T cells and peripheral blood mononuclear cell interleukin (IL)-10 secretion in humans.

- $B$ infantis-stimulated human dendritic cells induce Foxp3 and IL-10 secreting T cells.

- Dendritic cell subsets use different patternrecognition receptors to induce $\mathrm{T}$ regulatory cells.

- Dendritic cell metabolic responses (ie, retinoic acid and tryptophan metabolism) are essential.

How might it impact on clinical practice in the foreseeable future?

- $B$ infantis will be included in interventional approaches to confer maximum tolerogenic immunomodulatory activity in the gut in order to protect against gastrointestinal inflammatory processes.

comparison with pathogens to ensure tolerance and protective immunity, respectively. A characteristic feature of mucosal tolerance is the induction and expansion of Foxp3 T regulatory cells, which limit excessive proinflammatory responses. ${ }^{4}{ }^{5} \mathrm{We}$ and others have identified specific microbes, which selectively promote Foxp3 polarisation within the mucosa of mice. ${ }^{6-10}$ In addition, recent studies in patients with inflammatory diseases (ulcerative colitis and allergy) suggest that feeding with specific therapeutic microbes can increase the proportion of CD25 $5^{\text {high }}$ T cells. ${ }^{11} 12$ However, the in vivo mechanisms underpinning the microbiota-associated 
influence on T regulatory cells are not well understood and it is not clear whether the mechanistic results obtained in the murine system are also applicable to humans.

Within the gastrointestinal mucosa, a number of cell types including epithelial cells, intraepithelial lymphocytes, lamina propria macrophages and dendritic cells are required to maintain intestinal homoeostasis and tolerance. ${ }^{13}$ In particular, both myeloid (mDCs) and plasmacytoid dendritic cells (pDCs) are in close contact with microbes and are responsible for presenting microbial and dietary antigens to the adaptive immune system, thereby influencing polarisation of the adaptive response via cytokine and metabolite production. ${ }^{14-18}$ Thus, the decision to induce Foxp3 $\mathrm{T}$ cells is significantly influenced by activation of DC pattern-recognition receptors (PRRs), which programme DC gene expression and subsequent $\mathrm{T}$ cell polarisation. ${ }^{19}$ Coordination between PRR signalling pathways is important for the induction of the appropriate DC and T cell response. For example, Toll-like receptor-2 (TLR-2) recognition of zymosan results in the secretion of retinoic acid and interleukin (IL)-10 leading to Foxp3 induction, while dectin- 1 activation by zymosan leads to IL-23 secretion and Th17 induction. ${ }^{20}$ In addition, TLR-2 activation was shown to inhibit TLR-3-associated inflammatory responses within the skin in a TRAF-1-dependent mechanism. ${ }^{21}$ Furthermore, DC subsets may use different molecular mechanisms to cooperate in the induction of $T$ regulatory cells. ${ }^{22} 23$

Bifidobacterium longum subspecies infantis 35624 ( $B$ infantis) is a commensal microbe originally isolated from the human gastrointestinal mucosa and has been extensively studied for its ability to regulate inflammatory responses, both in mice and humans. ${ }^{6}{ }^{24-30}$ We selected this bacterium as a likely candidate for the ability to induce Foxp3 T regulatory cells in humans. Our results demonstrate that significantly elevated IL-10 responses and Foxp3 expression occur in humans after in vivo exposure to $B$ infantis by oral consumption. In addition, we provide evidence for the potential cellular and molecular mechanisms underpinning this regulatory effect as both human $\mathrm{mDCs}$ and $\mathrm{pDCs}$ specifically induce Foxp3+CD4+ cells following in vitro incubation with $B$ infantis. However, the mechanisms of Foxp3 induction differ for $\mathrm{mDCs}$ and $\mathrm{pDCs}$. Induction of Foxp3 T cells by $\mathrm{mDCs}$ is TLR-2-, DC-SIGN- and retinoic acid-dependent, whereas induction of Foxp3 cells by pDCs requires indoleamine 2,3-dioxygenase (IDO).

\section{MATERIALS AND METHODS Human studies}

Two healthy human volunteer studies were performed and both were approved by the clinical research ethics committee of the Cork Teaching Hospitals, Ireland. Each potentially eligible healthy adult volunteer was evaluated by a full clinical history review, physical examination, haematological and serum chemistry analysis. Clinically significant findings in any of the evaluation parameters led to the exclusion of that volunteer. In the first study, healthy adults were randomised to receive $B$ infantis $\left(\mathrm{n}=10 ; 1 \times 10^{9}\right.$ live bacteria per day) or placebo ( $\mathrm{n}=12$; cryoprotect with no bacteria) for 8 weeks. All investigators, as well as the volunteers, remained blinded to the randomisation process until completion of the study. The second study was conducted with healthy adults fulfilling the same selection criteria as outlined above but in this study all subjects were fed $B$ infantis ( $\mathrm{n}=17 ; 1 \times 10^{9}$ live bacteria per day) for 8 weeks.

Peripheral blood mononuclear cells (PBMCs) were isolated on day -1 (before feeding) and day 56 (after feeding). Freshly isolated PBMCs were stained with monoclonal antibodies to CD4-PerCP, CD25-APC, ICOS-PE, CTLA-4-PE and Foxp3-Alexa
Fluor 488 (eBioscience, San Diego, California, USA). Duplicate samples were evaluated using a FACSCalibur (Becton Dickinson, Oxford, England) and analysis was carried out using the BD CellQuest software. In addition, $1 \times 10^{6}$ cells $/ \mathrm{ml} \mathrm{PBMCs} \mathrm{were}$ stimulated with anti-CD3 $(5 \mu \mathrm{g} / \mathrm{ml})$ and anti-CD28 $(5 \mu \mathrm{g} / \mathrm{ml})$ antibodies for $48 \mathrm{~h}$. PBMC supernatants were analysed for cytokine levels simultaneously using the MesoScale Discovery multiplex platform.

\section{Dendritic cell isolation and culture conditions}

Dendritic cell experiments were performed using cells isolated from naïve (ie, non- $B$ infantis fed) healthy volunteers. Human peripheral blood monocytes were obtained using CD14 positive isolation with the MACS system (Miltenyi Biotec, Bergisch Gladbach, Germany). Cells were cultured in cRPMI media (Invitrogen, Carlsbad, USA) with 1000 U/ml IL-4 (Novartis, Basel, Switzerland) and $1000 \mathrm{U} / \mathrm{ml}$ granulocyte-macrophage colony-stimulating factor (PeproTech, London, UK) for 5 days in order to generate monocyte-derived dendritic cells (MDDCs). Peripheral blood mDCs were isolated following CD3, CD14 and CD19 depletion and CD1c positive selection with the MACS system. Peripheral blood pDCs were positively enriched using CD304 isolation with the MACS system. In addition, flow cytometric sorting with anti-CD123 FITC (Miltenyi Biotec) yielded a highly purified pDC population. Peripheral blood CD4 $\mathrm{T}$ cells were isolated by negative selection with the MACS system. MDDCs, $\mathrm{mDCs}$ and $\mathrm{pDCs}$ were routinely cultured in cRPMI medium and stimulated with bacterial strains or remained unstimulated. For blocking experiments, dendritic cells were preincubated for $30 \mathrm{~min}$ with control oligonucleotide GCTAGATGTTAGCGT, TLR9 antagonist oligonucleotide TTTAGGGTTAGGGTTAGGGTTAGGG (Microsynth, Balgach, Switzerland) or the blocking monoclonal antibodies: anti-TLR2 (gift from C Kirschning, Munich, Germany), anti-DC-SIGN (AZDN1, Beckman Coulter, Brea, USA) and IgG2B control antibody (R\&D Systems Europe, Abingdon, UK). Cytokine secretion was quantified by Bio-Plex multiplex suspension array (Bio-Rad Laboratories, Hercules, USA).

\section{Bacterial labelling and visualisation of cell binding}

$B$ infantis was stained with carboxyfluorescein diacetate, carboxyfluorescein succinimidyl ester (CFSE) (Invitrogen) while MDDCs were co-stained with anti-CD11c PE-Cy5 (BD Pharmingen, Franklin Lakes, USA). MDDCs were visualised at multiple time points using multispectral imaging flow cytometry Image Stream $\mathrm{X}$ (Amnis Corporation, Seattle, USA) and images were analysed using IDEAS software (Amnis Corporation). CD80 FITC and CD86 PE (BD Pharmingen) expression was evaluated by flow cytometry (Gallios system, Beckman Coulter, Nyon, Switzerland). MDDCs were incubated with CFSE-stained $B$ infantis for $16 \mathrm{~h}$, and $B$ infantis localisation within activated lysosomes was determined by staining MDDCs with Lysotracker red DND-99 (Invitrogen) and DAPI in ProLong Gold antifade reagent (Invitrogen). Slides were analysed by confocal microscopy.

\section{RALDH assessment}

MDDCs, mDCs and pDCs were stimulated with bacteria (with or without inhibitors) for $16-24 \mathrm{~h}$ in cRPMI. RALDH activity was determined using the ALDEFLOUR kit (Aldagen, Durham, USA) and positive cells were visualised by flow cytometry. In representative experiments, MDDCs were incubated with PKH26-stained $B$ infantis in order to co-localise bacterial binding and RALDH induction. 


\section{HEK-293 TLR-2 cells}

HEK-Blue hTLR-2 cells and HEK-Blue Null1 cells (Invivogen, San Diego, USA) were stimulated with $B$ infantis for $24 \mathrm{~h}$. Both cell lines express the NF-KB-inducible secreted embryonic alkaline phosphatase reporter. The TLR-2 ligand Pam3CSK4 $(2.5 \mu \mathrm{g} / \mathrm{ml}$, Calbiochem, Merck KGaA, Darmstadt, Germany) was used as a positive control.

\section{Dendritic cell T cell co-cultures}

Dendritic cells were stimulated for $4 \mathrm{~h}$ with test bacterial strains and co-cultured with autologous CD4 $\mathrm{T}$ cells at a 1:40 ratio (mDCs) or a 1:20 ratio (MDDCs and $\mathrm{pDCs}$ ) in AIM-V media (Invitrogen). After 5 days, cells were re-stimulated with antiCD28 (generated in house), anti-CD3 (Orthoclone OKT3, Janssen-Cilag) and anti-CD2 (Sanquin, Amsterdam, Netherlands). Two days later lymphocytes were permeabilised and stained for CD4, CD25 and Foxp3 (eBioscience). Citral (Sigma-Aldrich, St Louis, USA), LE135 (Tocris Bioscience, Bristol, UK) and 1-methyl-L-tryptophan (Sigma-Aldrich) were added as RALDH2 and IDO inhibitors, respectively.

\section{Statistical analysis}

Wilcoxon matched-pairs test or Student $t$ test were used to evaluate the effect of inhibitors on in vitro activated dendritic cells and dendritic cell-T cell co-cultures. In addition, D'Agostino and Pearson normality tests were performed on the cytokine and flow cytometric data from the human volunteer studies. Measurements before and after feeding were assessed using the Student $t$ test for paired data while comparisons between placebo and treatment groups were performed using a Student t test (two-tailed) and Mann-Whitney test. All data analysis was carried out using GraphPad Prism software.

\section{RESULTS}

Feeding of healthy human volunteers with $B$ infantis is associated with induction of IL-10 and Foxp3 in vivo

Healthy human volunteers were fed $B$ infantis or placebo for 8 weeks. PBMCs were isolated before and after the feeding period. $B$ infantis feeding was associated with significantly enhanced IL-10 levels in anti-CD3/CD28 stimulated PBMCs
Figure 1 Bifidobacterium infantis feeding in healthy humans is associated with increased secretion of interleukin (IL)-10. (A). In vitro stimulated peripheral blood mononuclear cells displayed increased secretion of IL-10 following $B$ infantis oral intake $(\mathrm{n}=10$; $\mathrm{p}=0.01$ ) for 8 weeks which was not seen in the placebo group $(n=12$; $p=0.64)$. (B). The increased secretion of IL-10 in healthy human volunteers following $B$ infantis feeding was repeated in a second study $(n=17)$. (C). While in vitro anti-CD3/CD28-stimulated peripheral blood mononuclear cells from $B$ infantis-fed volunteers displayed increased secretion of IL-10 $(p<0.001)$, there was no change in $\mathrm{LL}-2(\mathrm{p}=0.33)$, IL-12p70 ( $p=0.26)$, tumour necrosis factor (TNF) $\alpha(p=0.42)$ or interferon $($ IFN $) \gamma(p=0.56)$ secretion. ${ }^{*} p<0.05$ versus placebo; ${ }^{*}{ }_{1} \mathrm{p}<0.01$ versus pretreatment (week 0 ).
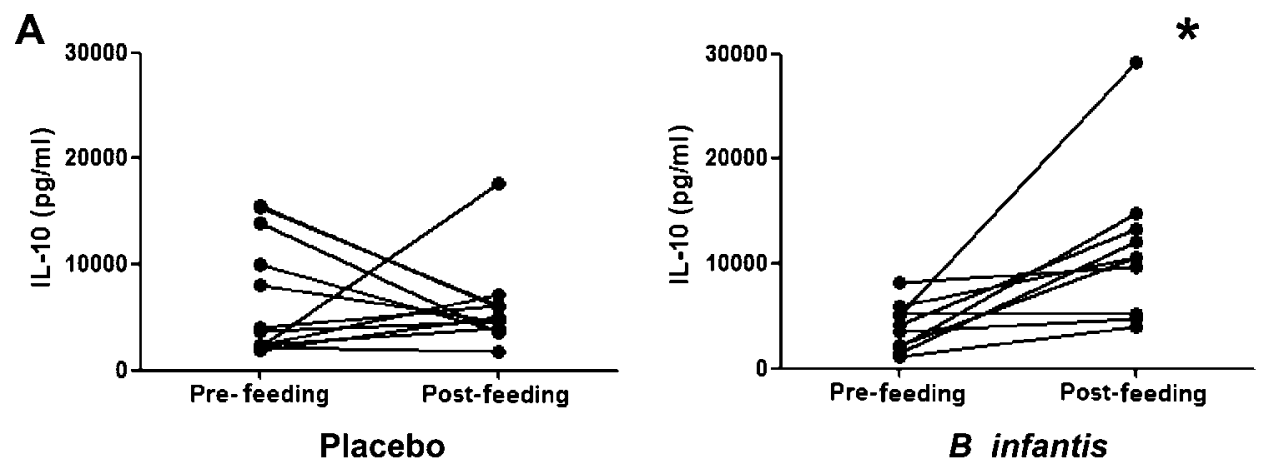

B
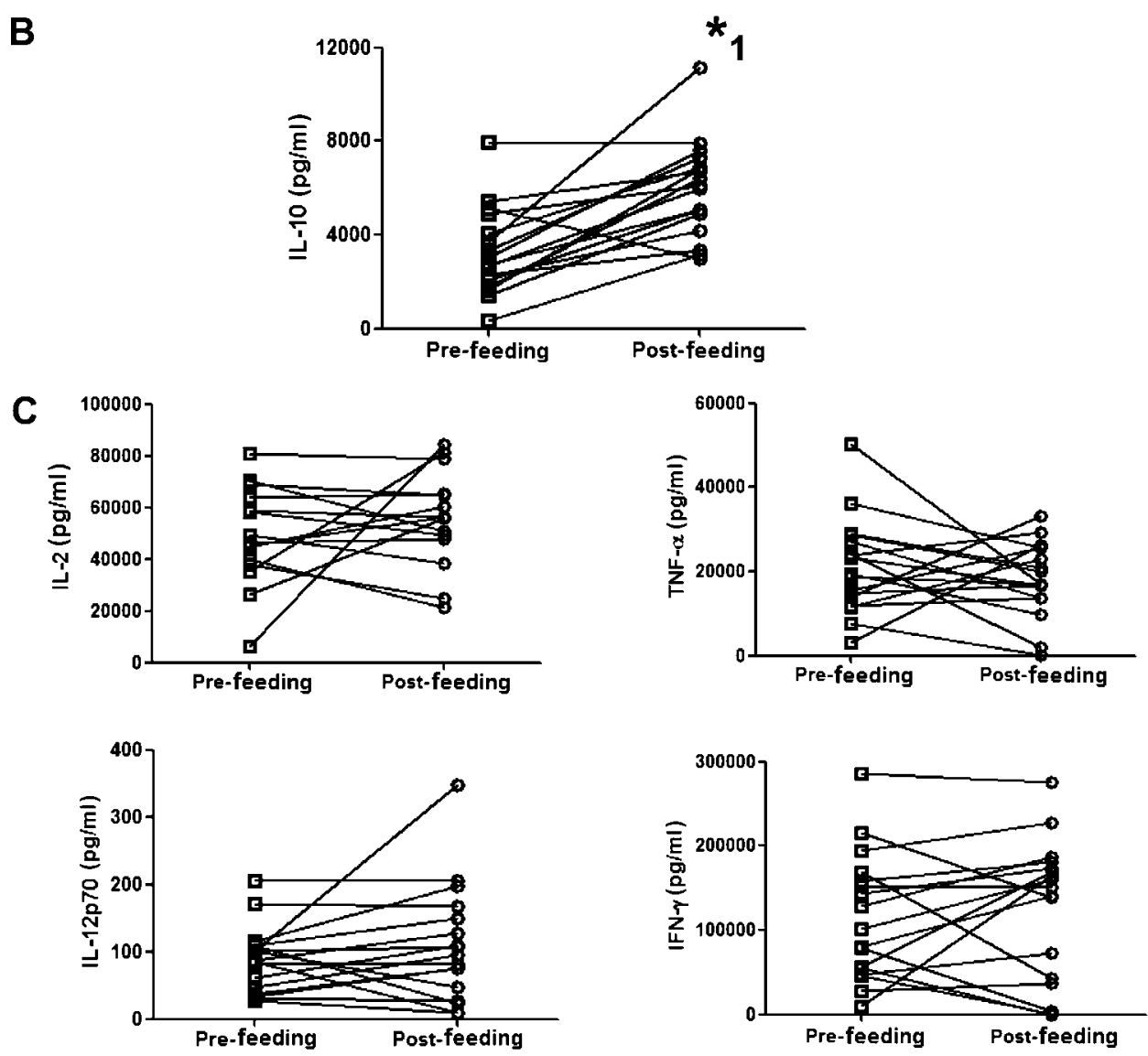
$(p=0.01)$, while IL-10 levels in the placebo group did not change $(p=0.64$; figure 1A). We performed a second study with greater numbers of healthy human volunteers, in which we reassessed PBMC IL-10 levels as before and in addition, we quantified Foxp3 expression. We again observed the same increase in
anti-CD3/CD28 stimulated PBMC secretion of IL-10 $(\mathrm{p}<0.001$ figure $1 \mathrm{~B})$, while we observed no difference in anti-CD3/CD28 stimulated IL-2, IL-12p70, tumour necrosis factor (TNF) $\alpha$ or interferon (IFN) $\gamma$ secretion (figure 1C). In addition, the percentage of peripheral blood CD4 $\mathrm{T}$ cells that express Foxp3
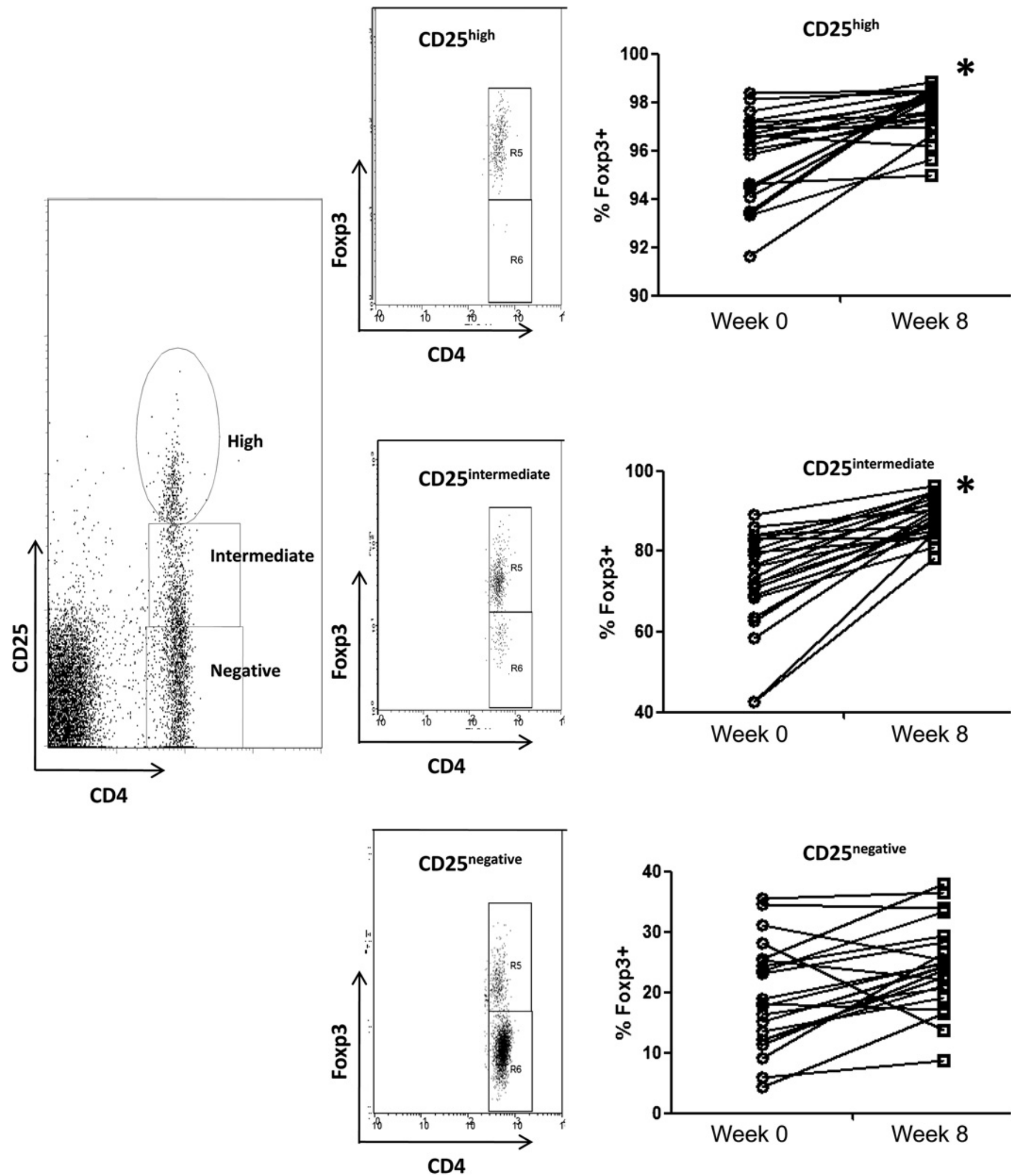

Figure 2 Bifidobacterium infantis feeding in healthy humans is associated with increased expression of Foxp3. Foxp3 expression was significantly increased in $\mathrm{CD} 25^{\text {high }}$ and $\mathrm{CD} 25^{\text {intermediate }} \mathrm{CD} 4 \mathrm{~T}$ cells, but not $\mathrm{CD} 25^{\text {negative }} \mathrm{CD} 4+\mathrm{T}$ cells, following $B$ infantis feeding. ${ }^{*} \mathrm{p}<0.01$ versus pretreatment (week 0 ). 
was significantly increased following $B$ infantis feeding (week $0 \mathrm{CD} 4+$ Foxp3 + cells $=8.14 \pm 0.25 \%$ vs week $8 \mathrm{CD} 4+$ Foxp3 + cells $=9.19 \pm 0.34 \% ; p=0.003)$. The increased expression of Foxp3 was seen in the CD25 $5^{\text {high }}$ and CD2 $5^{\text {intermediate }}$ CD4 T cells, but not in the CD25- T cells (figure 2). The CD4+CD25+Foxp3+ cells displayed a more pronounced regulatory phenotype after $B$ infantis administration as inducible T-cell costimulator (ICOS) was expressed by significantly more of these cells at week 8 (figure 3). Cytotoxic T-lymphocyte antigen 4 (CTLA-4) expression also increased but the difference did not reach statistical significance (figure $3, p=0.06$ ).

\section{$B$ infantis induces DC maturation and regulatory activity}

In order to understand how $B$ infantis induced IL-10 polarisation and expression of Foxp3 cells in humans, we focused on DCs as the potential cellular mediators, which could acquire $B$ infantis in vivo within the mucosa and secrete immunoregulatory factors that induce T cell polarisation. Human MDDCs, directly isolated human $\mathrm{mDCs}$ and directly isolated human $\mathrm{pDCs}$ were shown, in vitro, to bind $B$ infantis (figure $4 \mathrm{~A}, \mathrm{~B}$ ). In addition, MDDCs were able to internalise this microbe (figure $4 \mathrm{C}$ ). A dose-dependent maturation of MDDCs was evident from the increased expression of CD80 and CD86 (figure 4D). A similar increase in myeloid dendritic cell ( $\mathrm{mDC}$ ) costimulatory molecule expression was seen (results not shown).

$B$ infantis stimulated MDDCs, $\mathrm{mDC}$ and $\mathrm{pDCs}$ secreted IL10 , but not IL-12p70, in response to this bacterium (figure $5 \mathrm{~A}-\mathrm{C})$. This was not seen with all Bifidobacteria as MDDCs secreted IL-12p70, when co-incubated with other unrelated Bifidobacterial strains and pathogenic bacteria such as
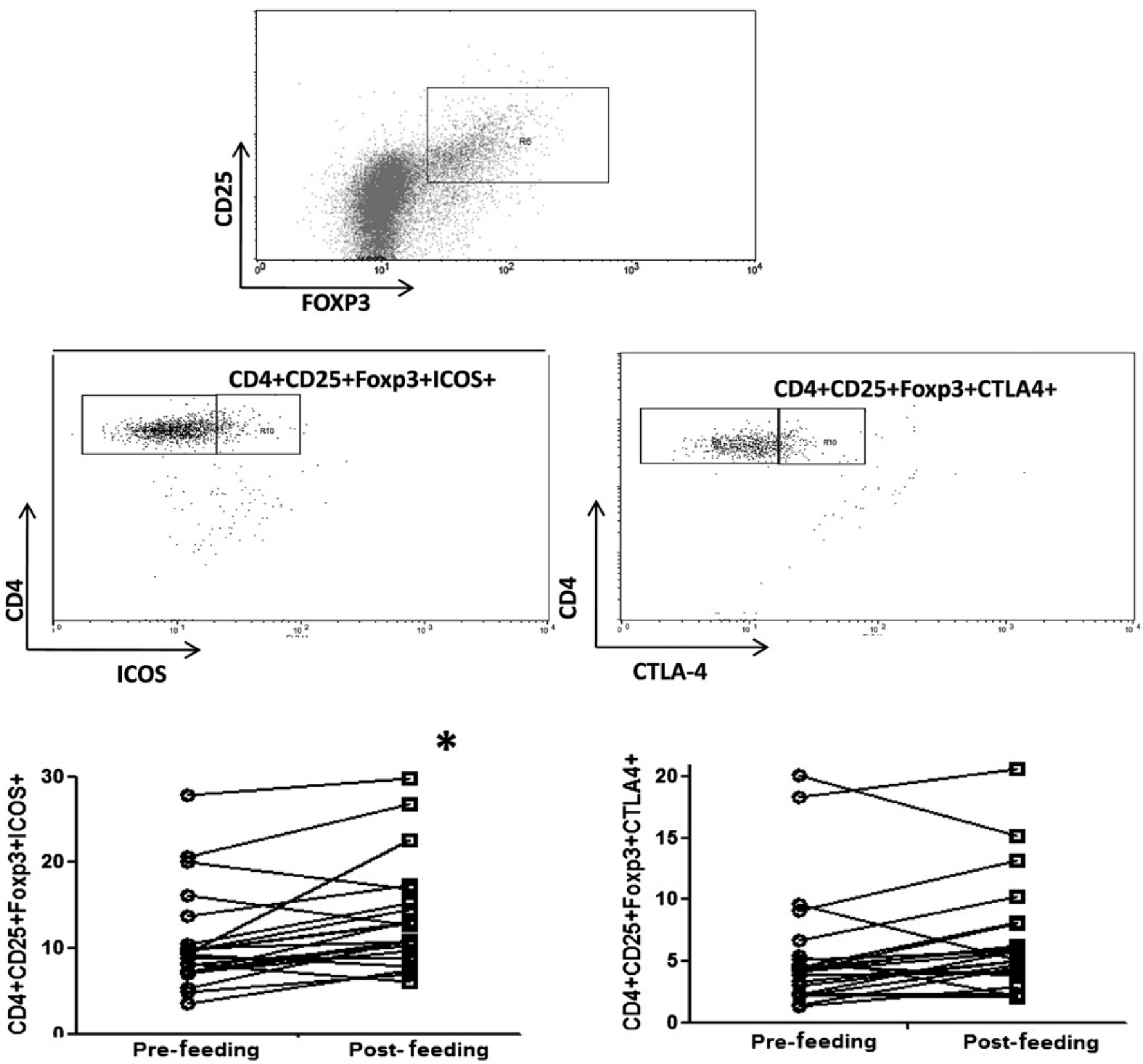

Figure 3 Bifidobacterium infantis feeding promotes expression of activation markers on Foxp3 T cells. Following feeding with $B$ infantis for 8 weeks, Foxp3 T cells from peripheral blood displayed significantly increased expression of inducible costimulator (ICOS) with a trend towards increased expression of cytotoxic T-lymphocyte antigen 4 (CTLA-4; $p=0.06$ ). ${ }^{*} p<0.05$ versus pretreatment (week 0 ). 
Figure 4 Myeloid and plasmacytoid dendritic cells (mDCs, pDCs) bind and internalise Bifidobacterium infantis. DCs efficiently bind $B$ infantis as visualised by multispectral flow cytometry imaging (A) and flow cytometry. (B) $B$ infantis is carboxyfluorescein diacetate, succinimidyl ester (CFSE) labelled (green) while CD11c+ cells are Pc5 labelled (red). Approximately $25 \%-35 \%$ of monocyte-derived dendritic cells (MDDCs), $\mathrm{mDC}$ or $\mathrm{pDCs}$ bind $B$ infantis after $2 \mathrm{~h}$ incubation at $37^{\circ} \mathrm{C}$, regardless of the DC subset. (C) After $16 \mathrm{~h}$ coincubation, internalised $B$ infantis cells were co-localised within MDDCactivated lysosomes. Activated lysosomes are coloured red, $B$ infantis on the MDDC surface are coloured green (CFSE labelled) while $B$ infantis inside activated lysosomes are coloured orange (combined red and green). Approximately $30 \%$ of the MDDC population internalise $B$ infantis. (D) CD80 and CD86 are significantly upregulated on MDDCs when exposed to $B$ infantis in a dose-dependent manner. Lipopolysaccharide (LPS) was used as the positive control stimulus. Each illustration $(A-D)$ is a representative example of at least five independent experiments.

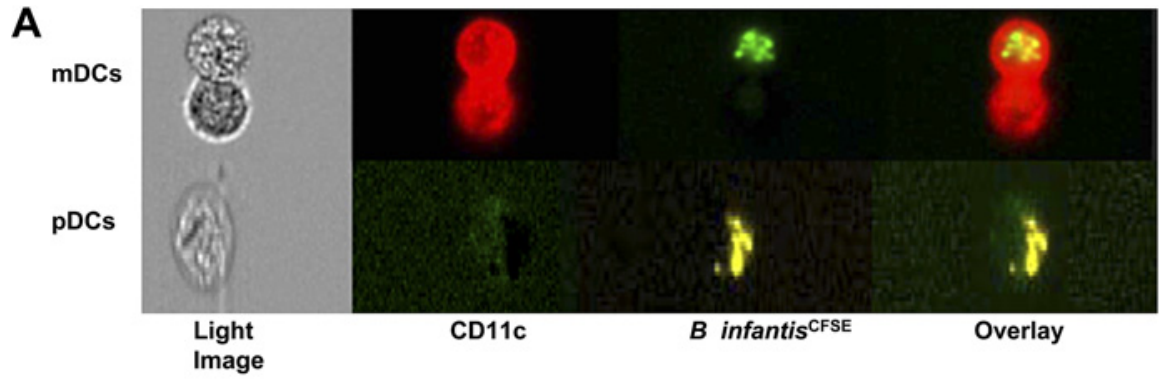

B

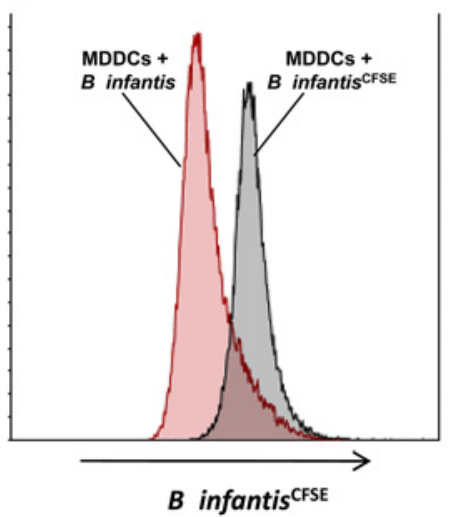

C
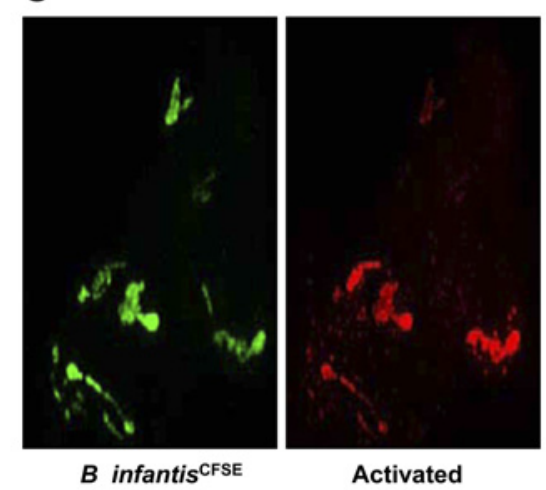

Activated lysosome DND-99

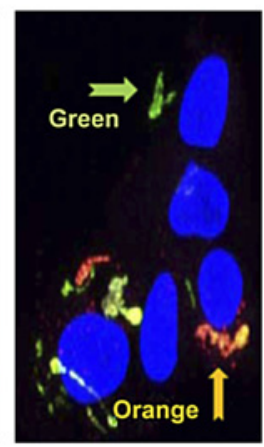

Overlay
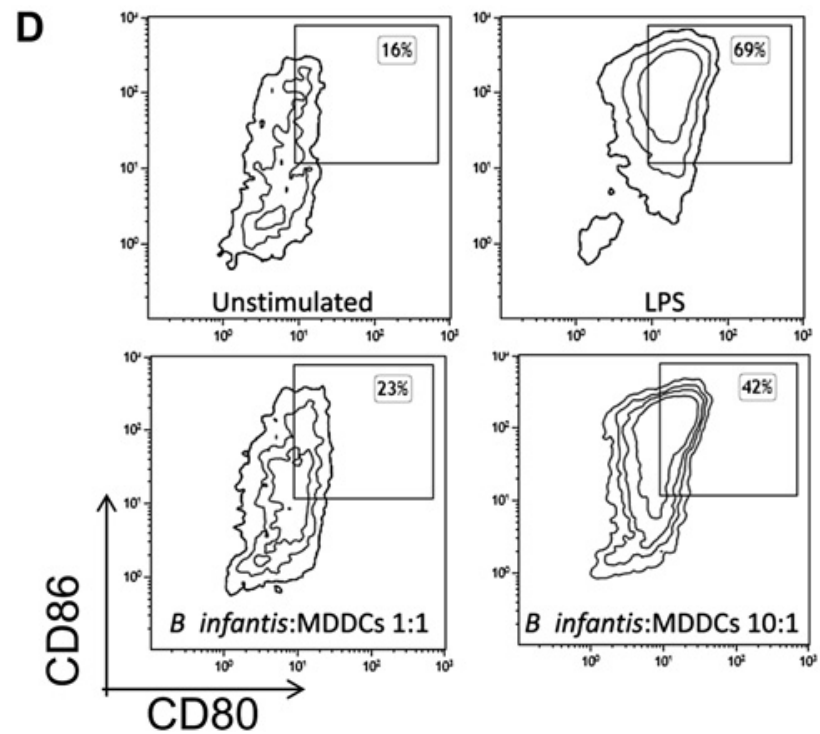

Streptococcus pyogenes, Staphyloccus aureus and Pseudomonas aeruginosa (supplementary figure S1). In addition to regulatory cytokines, $B$ infantis induced expression of the ALDH1A2 gene, which encodes for the enzyme retinaldehyde dehydrogenase 2 (RALDH2), in MDDCs and $\mathrm{mDCs}$ but not pDCs (figure $5 \mathrm{D}$ ). ALDH1A2 was induced quickly in MDDCs, while ALDH1A2 was induced at later time points in $\mathrm{mDCs} B$ infantis did not alter ALDH1A1 or ALDH1A3 gene expression in any DC subset (results not shown). The functional activity of RALDH2 was confirmed in MDDCs and $\mathrm{mDC}$ by flow cytometry (figure 5E). Inhibition of RALDH activity with diethylaminobenzaldehyde completely blocked the detection of BODIPY-aminoacetate-positive cells. However, pDCs did not upregulate RALDH activity with $B$ infantis incubation (figure 5E). MDDCs which had bound $B$ infantis, expressed a higher level of RALDH metabolic activity that MDDCs that had not captured $B$ infantis (supplementary figure S2A,B). In addition, the induction of RALDH was dose dependent and the optimal dose of approximately $5 \times 10^{6}$ bacterial cells (10:1 bacteria:MDDC cell number) was used in subsequent experiments (supplementary figure S2C). Another metabolic enzyme with regulatory activity is IDO. $B$ infantis induced IDO gene expression in MDDCs, $\mathrm{mDCs}$ and $\mathrm{pDCs}$, with relatively higher levels of expression by pDCs (figure $5 \mathrm{~F}$ ). The kinetics of IDO induction was different for each DC subset. Expression of MDDCs slowly increased over time, while $\mathrm{mDC}$ reached maximal expression levels at $6 \mathrm{~h}$ and expression was maintained over time. Similarly, expression of pDCs IDO peaked at $6 \mathrm{~h}$ but decreased rapidly thereafter.

The induction of $A L D H 1 A 2$ in MDDCs by $B$ infantis 35624 was not seen with the other Bifidobacterial strains that were examined (supplementary figure S3A), while IDO gene expression was enhanced by lipopolysaccharide and all three Bifidobacterial strains (supplementary figure S3B). 


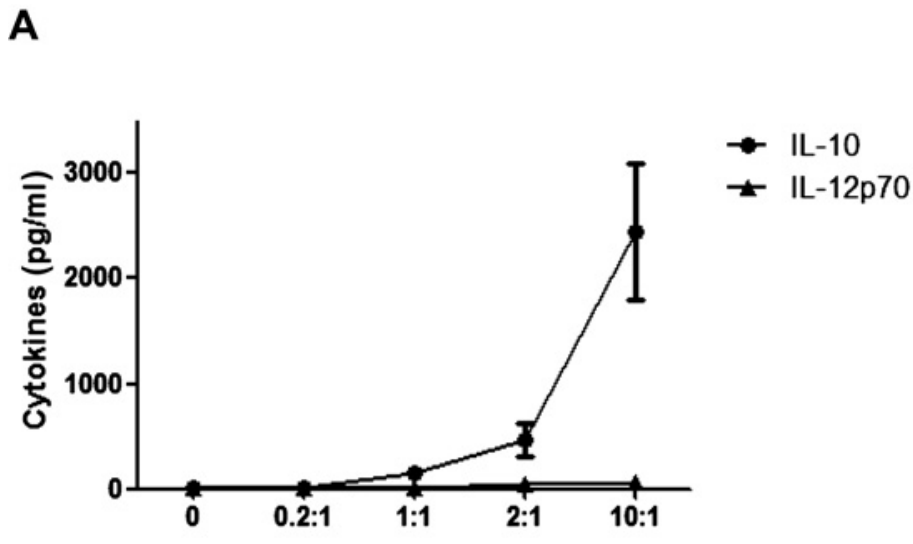

Bacteria:MDDC cell number
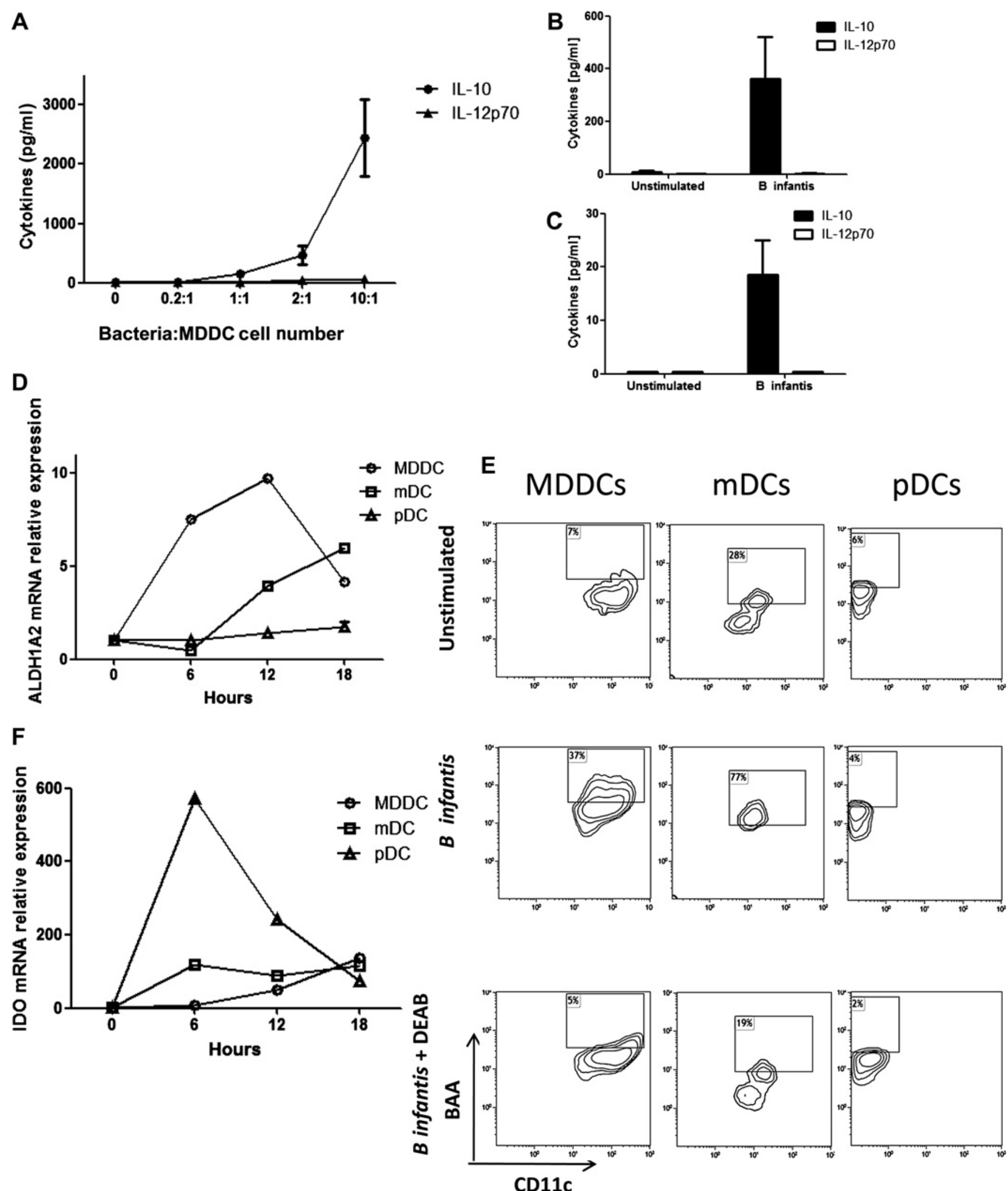

$\mathbf{E}$

MDDCs

mDCs

pDCs
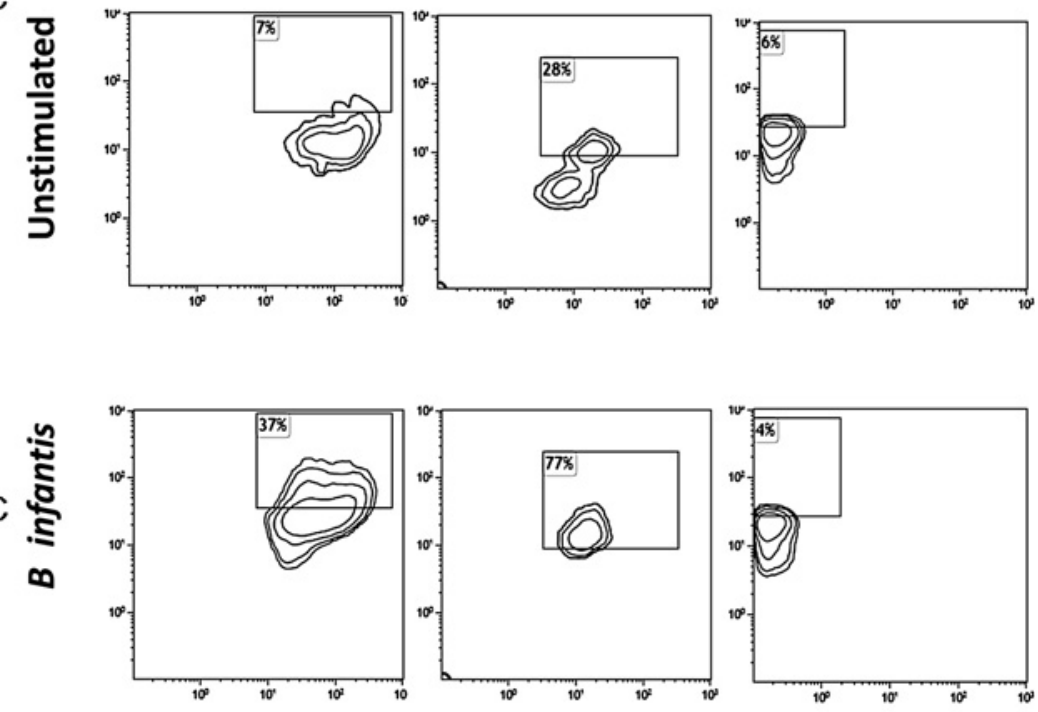

CD11c

Figure 5 Bifidobacterium infantis induces a regulatory dendritic cell phenotype. IL-10 secretion by monocyte-derived dendritic cells (MDDCs) in response to $B$ infantis increased in a dose-dependent manner, while interleukin (IL)-12p70 levels remained low ( $A$; mean $\pm S E, n=5$ donors). Similarly, directly isolated myeloid DCs ((mDCs) $\mathrm{B} ; \mathrm{n}=3$ donors) and plasmacytoid DCs ((pDCs) C; $\mathrm{n}=3$ donors) secreted IL-10, but not IL-12p70 in response to $B$ infantis. (D) ALDH1A2 gene expression (encoding the retinaldehyde dehydrogenase 2 (RALDH2) enzyme) was quantified every $6 \mathrm{~h}$, until $18 \mathrm{~h}$, after $B$ infantis stimulation of MDDCs, mDCs and pDCs. (E) The functional activity of RALDH was determined by detection of BODIPY-aminoacetate (BAA)positive MDDCs and mDCs, but not pDCs, exposed to $B$ infantis for $24 \mathrm{~h}$. The RALDH inhibitor diethylaminobenzaldehyde (DEAB) blocked the conversion of the substrate. Gene expression of the metabolic enzyme indoleamine 2,3-dioxygenase (IDO) was seen in MDDCs, mDCs and pDC (F). (D-F) All experiments were repeated independently for at least four donors. 


\section{$B$ infantis activation of DCs is PRR dependent}

In order to better understand the molecular basis for $B$ infantisinduced DC immunoregulatory responses, we next examined the role played by a range of PRRs. MDDCs express high levels of Dectin-1; however, no significant differences in cytokine production were seen for IL-10 $(1626 \pm 453 \mathrm{pg} / \mathrm{ml}$ vs $1522 \pm 418 \mathrm{pg} / \mathrm{ml})$ or IL- $12 \mathrm{p} 70(12.5 \pm 4.8 \mathrm{pg} / \mathrm{ml}$ vs $11.9 \pm 4.1 \mathrm{pg} /$ $\mathrm{ml}$ ) secretion with Dectin-1 inhibition. Blockade of MDDC TLR-2 resulted in significantly decreased secretion of IL-10 and significantly increased secretion of IL-12p70 and TNFa (figure 6A). Inhibition of MDDC DC-SIGN did not significantly alter IL-10 or IL-12 secretion, although TNF $\alpha$ secretion was significantly increased (figure 6A). Blockade of mDC TLR-2 or DC-SIGN resulted in significantly reduced IL-10 secretion associated with increased secretion of $\mathrm{TNF} \alpha$ (figure $6 \mathrm{~B}$ ). In order to confirm that TLR-2 induced an intracellular signalling cascade in response to $B$ infantis, we examined HEK-293 cells, which were engineered to express TLR-2. HEK-293 ${ }^{\text {TLR-2+ }}$ cells responded to $B$ infantis as demonstrated by an increase in NF- $\mathrm{KB}$ activation and IL-8 secretion (supplementary figure S4). In contrast, NF-KB activation and IL-8 secretion did not increase over baseline levels in HEK ${ }^{\text {TLR-2- }}$ cells. In addition, preincubation of HEK-293 $3^{\text {TLR-2+ }}$ cells with anti-TLR-2 blocking antibody significantly reduced NF-KB activation and IL-8 secretion in response to $B$ infantis. TLR-2 can form heterodimers with either TLR-1 or TLR-6. Blocking TLR-1 had no effect on $B$ infantis-induced NF-KB activation or IL-10 secretion, while TLR-6 had a partial effect but not as significant as TLR-2 blockade (supplementary figure S5). The combination of antiTLR-2 and anti-TLR- 6 antibodies was the most effective in the inhibition of HEK-293 NF- $\kappa B$ activation and MDDC IL-10 secretion. TLR-2 was expressed by pDCs at a very low level and
Figure 6 Dendritic cell (DC) cytokine production is pattern-recognition receptor dependent. (A) $B$ infantis stimulated monocyte-derived dendritic cells were preincubated with blocking antibodies to Toll-like receptor-2 (TLR-2; $\mathrm{n}=9$ ) or DC-SIGN $(\mathrm{n}=8)$ and cytokine secretion was quantified after $24 \mathrm{~h}$ stimulation. Blocking of TLR-2

significantly reduced interleukin (IL)-10 secretion, which was accompanied by a significant increase in IL-12p70 and tumour necrosis factor (TNF) $\alpha$ secretion. Blocking of DC-SIGN had no impact on IL-10 or IL-12p70 secretion but did result in increased TNF $\alpha$ secretion. (B) Bifidobacterium infantis stimulated myeloid DCs were preincubated with blocking antibodies to TLR-2 $(n=3)$ or DC-SIGN $(n=3)$ and cytokine secretion was quantified after $24 \mathrm{~h}$ stimulation. Blocking of TLR-2 or DC-SIGN significantly reduced IL-10 secretion, which was accompanied by a significant increase in TNF $\alpha$ secretion. (C) IL-10 secretion by plasmacytoid DCs (pDCs) in response to $B$ infantis was unchanged between isotype control antibody (IC) and anti-TLR-2 blocking antibody treated cells $(n=4)$. (D) Preincubation of $\mathrm{pDC}$ with blocking TLR9-specific oligodeoxynucleotides (ODNs) significantly reduced $\mathrm{pDC}$ secretion of IL-10 in response to $B$ infantis stimulation. (E) IL-10, indoleamine 2,3-dioxygenase (IDO) and interferon (IFN) $\alpha$ gene expression were quantified in $\mathrm{pDCs}$ after stimulation with CpG or $B$ infantis for $0,6,12$ or $18 \mathrm{~h}$. ${ }^{*} p<0.01$ versus isotype control antibody or control ODN-treated DCs.
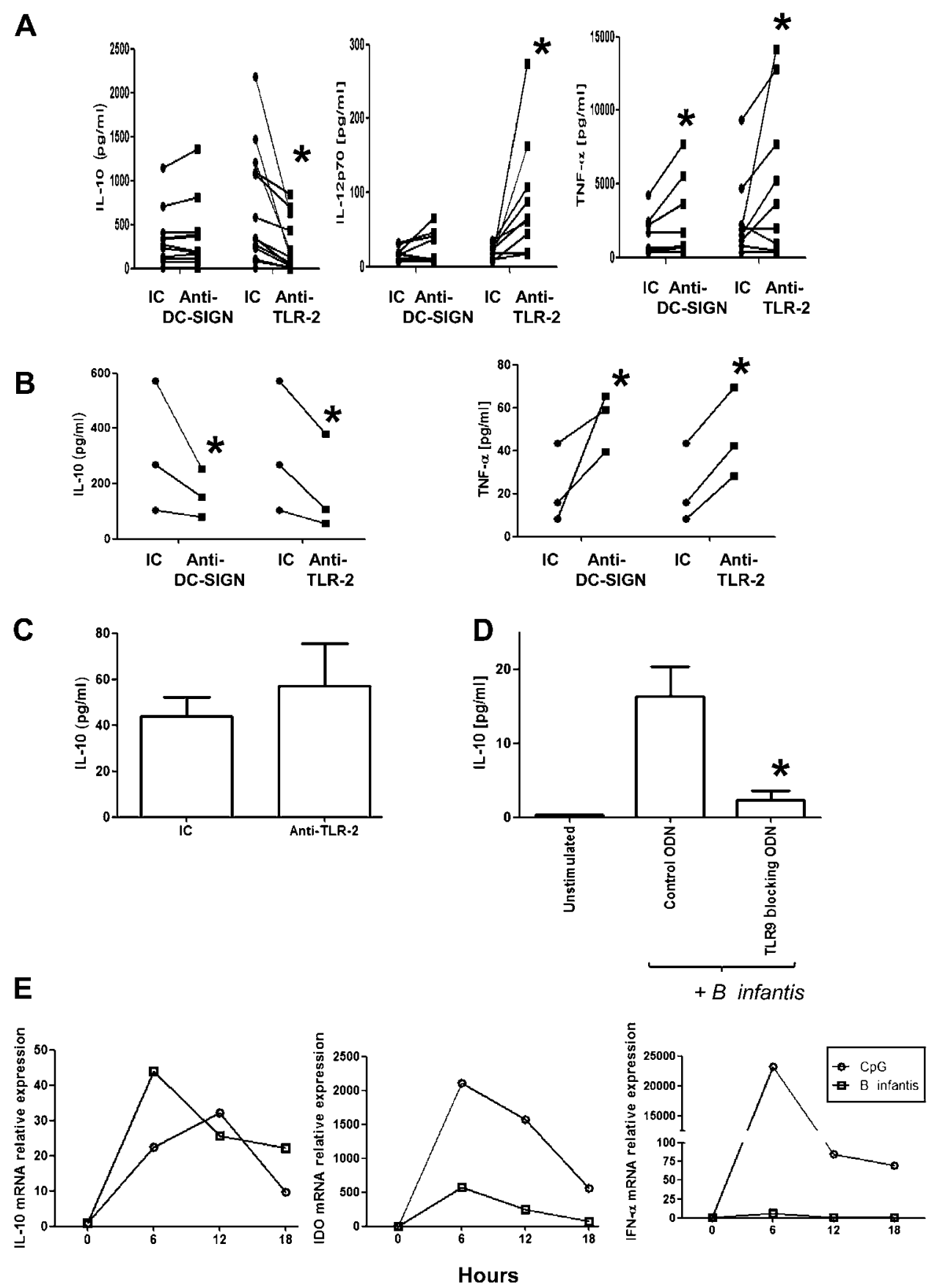
blocking experiments did not alter the pDC IL-10 response to $B$ infantis (figure 6C), while IL-12p70 was not detected. However, blockade of TLR-9 with an inhibitory oligonucleotide abolished the secretion of IL-10 by $\mathrm{pDCs}$ in response to $B$ infantis (figure 6D). In order to evaluate if $\mathrm{pDC}$ respond in a similar manner to specific TLR-9 agonists, pDCs were stimulated with $\mathrm{CpG}$ or $B$ infantis. Both $B$ infantis and $\mathrm{CpG}$ stimulation resulted in enhanced IL-10 and IDO gene expression, while IFN $\alpha$ gene expression was only increased after $\mathrm{CpG}$ stimulation (figure $6 \mathrm{E}$ ). MDDCs and mDCs express very low levels of TLR-9 and TLR-9 inhibitory oligonucleotides did not alter the MDDC response to $B$ infantis (results not shown).

In addition to cytokine production, the blocking of MDDC TLR-2 or DC-SIGN resulted in significant inhibition of retinoic acid metabolism in $B$ infantis-stimulated MDDCs and $\mathrm{mDCs}$ (figure 7A,B). Furthermore, specific activation of MDDC TLR-2 by Pam3CSK 4 induced retinoic acid metabolism, while stimulation of DC-SIGN by mannosylated lipoarabinomannan (ManLam) marginally increased retinoic acid metabolism (figure 7C). In contrast, specific activation of TLR-4 or TLR-5 by lipopolysaccharide or flagellin, respectively, did not induce retinoic acid metabolism in MDDCs (figure $7 \mathrm{C}$ ).

\section{$B$ infantis-stimulated DCs induce Foxp3 T cells}

As human DCs clearly secrete immunoregulatory mediators in response to $B$ infantis, we assessed whether these DCs could also induce Foxp3 expression by autologous T cells in vitro. MDDCs were incubated with $B$ infantis for $4 \mathrm{~h}$, washed and re-incubated with autologous CD4 cells for 5, 7 or 9 days. Peak induction of Foxp3, compared with unstimulated MDDCs was seen at 7 days with or without costimulation (supplementary figure S6A). In all subsequent experiments, DCs were co-cultured for 7 days with autologous CD4 T cells for assessment of Foxp3 induction. After incubation with $B$ infantis, MDDCs, $\mathrm{mDCs}$ and pDCs induced Foxp3 expression in cultures with CD4 T cells (figure 8A). In addition, enhanced IL-10 secretion was seen in the $B$ infantis-stimulated DC-T cell co-cultures, compared with T cells incubated with unstimulated DCs (figure 8B). Blocking TLR-2 significantly reduced IL-10 secretion from MDDC and $\mathrm{mDC}-\mathrm{T}$ cell co-cultures, with no effect on $\mathrm{pDC}-\mathrm{T}$ cell IL-10 secretion (figure 8C), while blocking TLR-9 significantly reduced $\mathrm{pDC}-\mathrm{T}$ cell secretion of IL-10 (figure 8D).

In order to determine which PRRs were responsible for the preferential induction of Foxp3 T cells, MDDCs were preincubated with anti-TLR-2 or anti-DC-SIGN blocking antibodies before $B$ infantis activation. A significant reduction in the CD4 +CD25+Foxp3+ population was seen when dendritic cell TLR2 responses were blocked. To a lesser extent, blockade of DCSIGN also significantly reduced CD4+CD25+Foxp3+ induction (figure 9A). No induction of T-bet or GATA-3 positive $\mathrm{T}$ cells over baseline was seen. However, incubation of MDDCs with other Bifidobacteria, which induced IL-12p70 secretion from dendritic cells, resulted in the induction of $\mathrm{T}$-bet $+\mathrm{T}$ cells (supplementary figure S6B). RALDH2 induction has been associated with the enhanced capacity of mucosal associated CD103+ dendritic cells to induce Foxp3 $\mathrm{T}$ cells. After inhibition of $\mathrm{MDDC}$ or $\mathrm{mDC}$ retinoic acid synthesis (using citral) or inhibition of $T$ cell retinoic acid receptor signalling (using LE135), the induction of Foxp3 T cells by $B$ infantis-stimulated MDDCs and mDCs was suppressed (figure 9B). Using the IDO inhibitor 1-methyl tryptophan, induction of Foxp3 T cells by $B$ infantisstimulated pDCs was inhibited (figure 9C). B infantis-stimulated MDDC induction of Foxp3 T cells was also partially reduced in the presence of 1-methyl tryptophan; however, the reduction
A
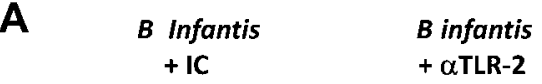

$+\alpha$ TLR-2

$B$ infantis
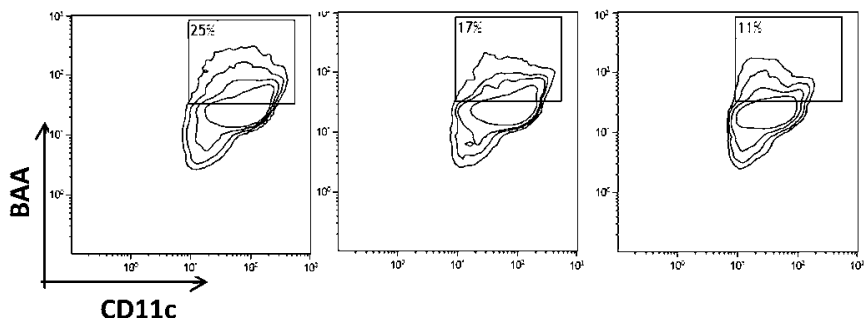

CD11c

B
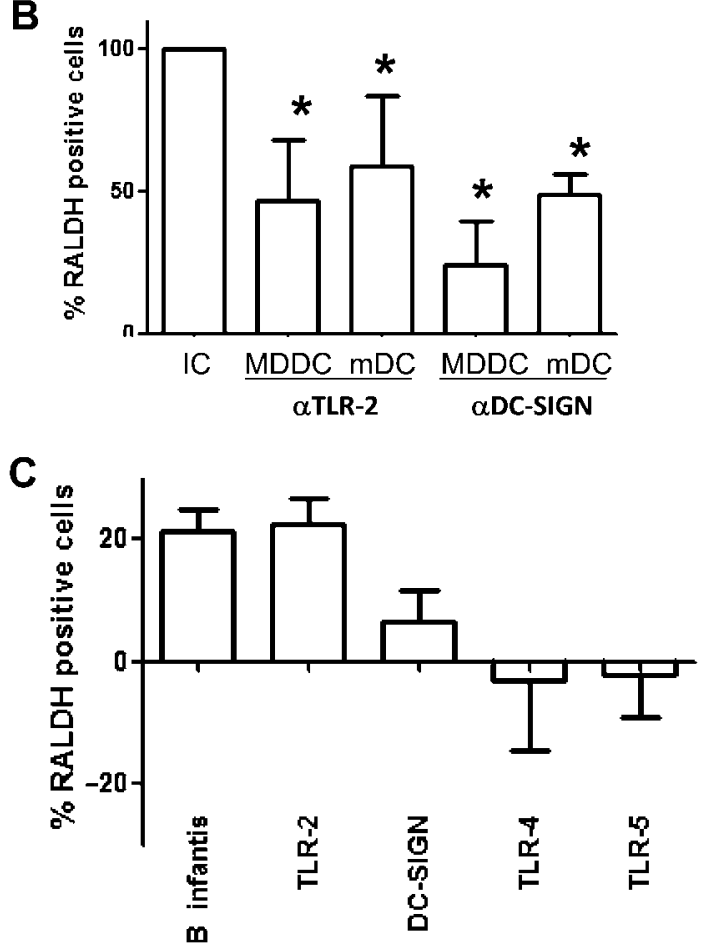

Figure 7 Toll-like receptor-2 (TLR-2) and DC-SIGN induce retinoic acid metabolism. (A) Blocking of either TLR-2 or DC-SIGN significantly attenuated retinaldehyde dehydrogenase 2 (RALDH2) induction by Bifidobacterium infantis-stimulated monocyte-derived dendritic cells (MDDCs). (B) The mean inhibition of RALDH activity by anti-TLR-2 or anti-DC-SIGN antibodies was equivalent for both MDDCs and myeloid dendritic cells (mDCs; $n=4$ donors). Isotype control antibodies (IC) were included in all experiments. (C) Stimulation of MDDCs with TLR-2 (Pam3CSK4) or DC-SIGN (ManLam)-specific ligands induced retinoic acid metabolism, while TLR-4 (lipopolysaccharide) or TLR-5 (flagellin) stimulation did not. ${ }^{*} p<0.05$ versus isotype control antibody. BAA, BODIPY-aminoacetate.

was not statistically significant and not as substantial as that seen for pDC-T cells (figure 9D).

\section{DISCUSSION}

Induction of Foxp3 $\mathrm{T}$ regulatory cells is a pivotal feature of mucosal immune tolerance. In this study, we show that oral feeding of healthy human volunteers with $B$ infantis results in increased numbers of Foxp3 $+\mathrm{CD} 4+\mathrm{T}$ cells within peripheral blood, which is associated with enhanced secretion of IL-10 following stimulation. In addition, our in vitro data support the hypothesis that DCs are responsible for the induction of Foxp3 cells. Both $\mathrm{mDC}$ and $\mathrm{pDC}$ subsets play a role, but recognise $B$ infantis via different PRRs. Induction of Foxp3 $\mathrm{T}$ cells by MDDCs and mDCs involves TLR-2, DC-SIGN and retinoic 

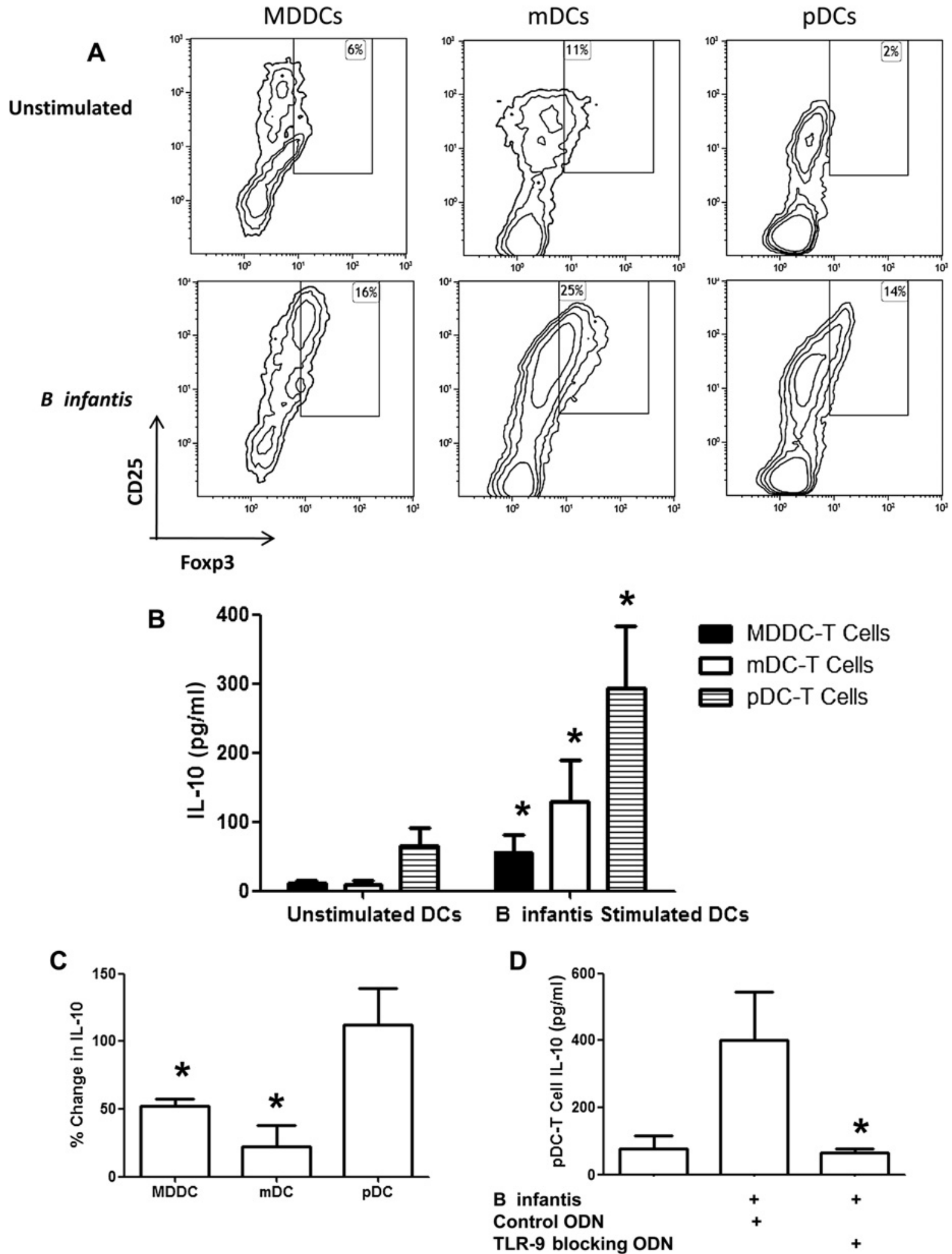

Figure 8 Bifidobacterium infantis stimulated dendritic cells induce Foxp3 T cells and interleukin (IL)-10 secretion. B infantis stimulated monocytederived dendritic cells (MDDCs), myeloid DCs (mDCs) or plasmacytoid DCs (pDCs) induce CD25+Foxp3+ T cells (A) associated with increased secretion of IL-10 ( $\mathrm{B}, \mathrm{n}=6$ donors) compared with unstimulated MDDC-, mDC- or pDC-T cell co-cultures. The flow cytometry density plots illustrated are from anti-CD2/CD3/CD28 restimulated T cell cultures. (C) Blocking Toll-like receptor-2 (TLR-2) significantly reduced IL-10 secretion from MDDC and $\mathrm{mDC}-\mathrm{T}$ cell co-cultures, but had no effect on $\mathrm{pDC}-\mathrm{T}$ cell IL-10 secretion $(\mathrm{n}=3$ donors). (D) Blocking TLR-9 significantly reduced pDC-T cell secretion of IL-10 $(n=4) .{ }^{*} p<0.05$ versus unstimulated cells. ODN, oligodeoxynucleotides.

acid metabolism. However, induction of Foxp3 T cells by pDCs is independent of TLR-2 and retinoic acid but requires IDO. To our knowledge this is the first report which demonstrates that human DC subsets use different pathways when exposed to a commensal microbe that enhances Foxp3 expression in autologous CD4 T lymphocytes. 
Figure 9 Toll-like receptor-2 (TLR-2), DC-SIGN, retinaldehyde dehydrogenase 2 (RALDH2) and indoleamine 2,3dioxygenase (IDO) are required for optimal induction of Foxp3 T cells. (A) Induction of Foxp3 T cells by Bifidobacterium infantis was blocked when monocyte-derived dendritic cells (MDDCs) were preincubated with antiTLR-2 or anti-DC-SIGN blocking antibodies. In contrast, there was no effect on T-bet or GATA-3 expression by $T$ cells (mean $\pm S E, n=4$ donors). (B) Inhibition of retinoic acid production (citral) or retinoic acid signalling (LE135) blocked the induction of Foxp3 cells by $B$ infantis-stimulated MDDCs and myeloid DCs (mDCs; $\mathrm{n}=3$ donors). (C) In addition, inhibition of pDC IDO activity by 1 -methyl tryptophan significantly attenuated the plasmacytoid $\mathrm{DC}(\mathrm{pDC})$ induction of Foxp3 T cells. (D) IDO inhibition partially reduced $B$ infantis-stimulated MDDC-T cell Foxp3 induction ( $\mathrm{n}=3$ donors) but not as significantly as IDO inhibition for pDC-T cell Foxp3 induction $(\mathrm{n}=5$ donors). ${ }^{*} \mathrm{p}<0.05$ versus unstimulated cells.

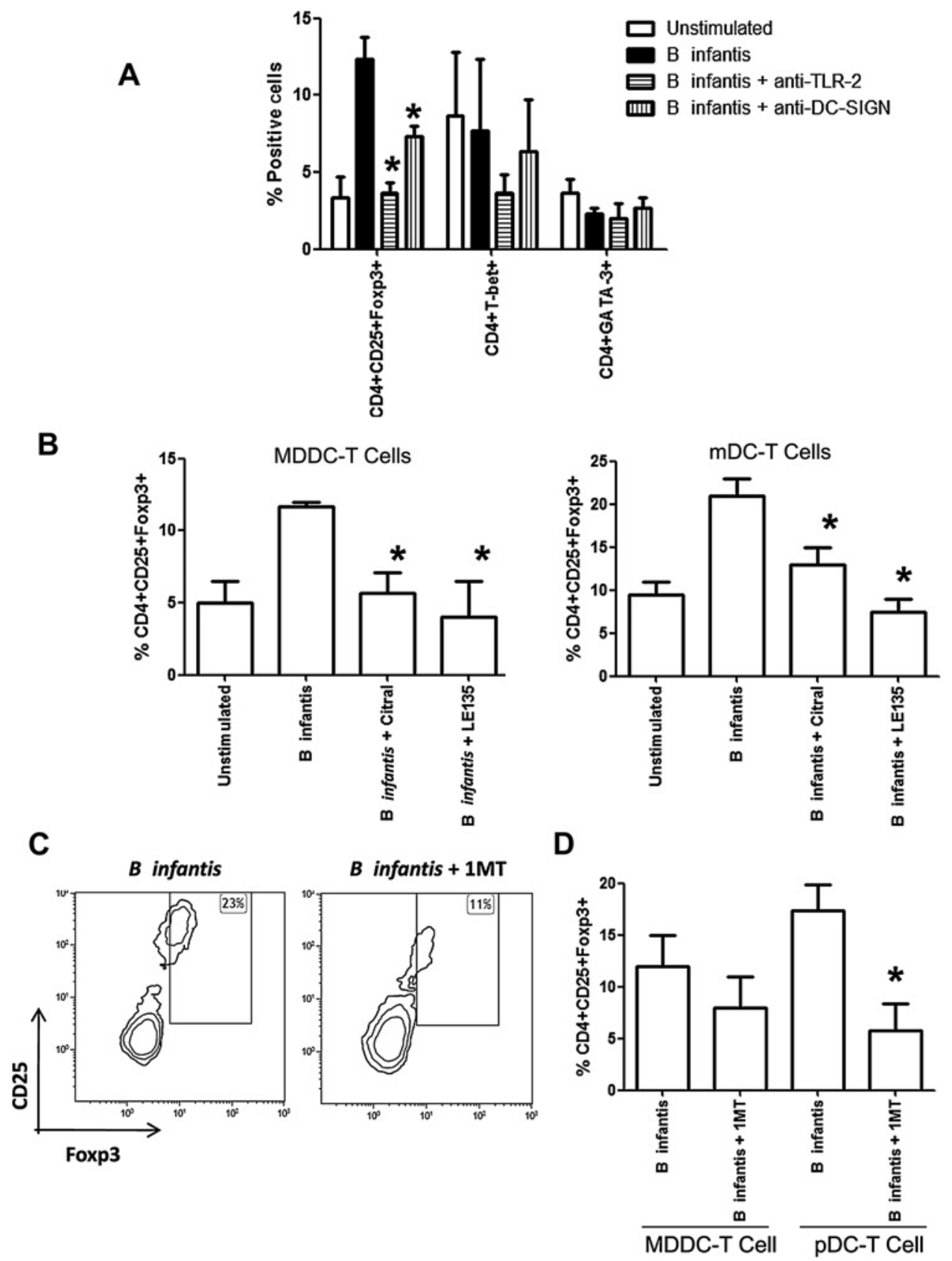

Human Foxp3 T cells are heterogeneous in phenotype and function, composing distinct, although related, subpopulations. Interestingly, enhanced expression of ICOS and CTLA-4 within the Foxp3 population suggests that $B$ infantis induces an effector Treg phenotype. ${ }^{31} B$ infantis feeding was associated with increased numbers of CD25 cells that expressed Foxp3 but there was no effect on Foxp3 expression within the CD25- population. One possible explanation is that Foxp3 can promote CD25 expression, and therefore $B$ infantis-associated induction of Foxp3 in CD25- cells leads to the expression of CD25 and their inclusion in the CD25 gate. ${ }^{32}$ Alternatively, $B$ infantis might not induce new CD25+Foxp3+ cells in vivo, but rather $B$ infantis might stabilise and promote expansion of the pre-existing CD25 +Foxp3+ population.

The default status of the gastrointestinal microenvironment is thought to favour induction of regulatory lymphocytes. For example, retinoic acid generation from vitamin A occurs in intestinal epithelial cells and specialised DC subsets, resulting in the conversion of naïve $\mathrm{T}$ cells into Foxp3 $\mathrm{T}$ regulatory cells. ${ }^{33}$ However, the gut-specific factors which promote vitamin A metabolism have been poorly described. We describe upregulation of the retinoic acid metabolising enzyme RALDH2 in mDCs, which are exposed to a single commensal strain. The retinoic acid response is functional and contributes to the induction of Foxp3 T cells. Thus, the presence of certain bacterial strains within the gastrointestinal tract may provide the necessary signals to gut-associated lymphoid tissue for the induction of enzymes, such as RALDH2, which are required for maintaining intestinal homoeostasis in a milieu of exogenous antigenic challenge. Surprisingly, tryptophan metabolism, but not retinoic acid metabolism, is required for the induction of Foxp3+CD4+ T cells by pDCs upon $B$ infantis exposure. These molecular mechanisms highlight an important link between diet, composition of the gastrointestinal microbiota and regulation of intestinal immune responses. Interestingly, recent findings by other investigators on microbiota-derived 
short-chain fatty acids suggest that we may have previously underestimated the importance of the relationship between diet and the microbiota. ${ }^{34}$ In addition, acetate production (following carbohydrate fermentation) by certain Bifidobacteria strains mediates protection from lethal Escherichia coli infection in mice. $^{35}$

Recent findings on the role of PRR signalling in mucosal homoeostasis have emphasised the delicate balance between different PRR functions, and shown that defective PRR signalling can result in inflammation. ${ }^{36}$ TLR-2 $\mathrm{KO}$ animals are more sensitive to dextran sodium sulphate-induced colitis, while TLR2 gene variants are associated with disease phenotype in patients with inflammatory bowel disease. Indeed, TLR-2 has been shown to promote Foxp3 expression in response to intestinal microbes in murine models. ${ }^{37} 38$ Our results show that inhibition of human $\mathrm{mDC}$ TLR-2/6 activation suppressed IL-10 secretion and Foxp3 induction, while enhancing secretion of IL-12p70 and TNF $\alpha$ following $B$ infantis exposure. DC-SIGN activation by $B$ infantis is required for full RALDH activity, and blocking DC-SIGN results in significantly fewer Foxp3+CD4+ T cells. Similarly, TLR-9 is required for pDC secretion of IL-10 in response to $B$ infantis. However, $\mathrm{CpG}$ stimulation of $\mathrm{pDCs}$ resulted in enhanced IFN $\alpha$ gene expression, which was not seen with $B$ infantis stimulation, suggesting that other molecular pathways distinct from those induced solely by TLR-9 are involved in the $\mathrm{pDC}$ response to this bacterium. These findings suggest that cross-talk between TLR-2/6, DC-SIGN, TLR-9 and other PRRs determines the innate and subsequent adaptive immune response to this commensal microbe. Appropriate PRR activation in vivo may promote immune homoeostatic mechanisms, which limit the activation of proinflammatory responses and protect mucosal tissue from injury. In addition, the involvement of multiple PRRs suggests that multiple bacterial components including lipoteichoic acids (TLR-2), polysaccharides (DC-SIGN) and DNA (TLR-9) are all important for optimal induction of the immune regulatory programme in vivo. Furthermore, a commensal microbe that expresses all of these regulatory factors may be more efficient in the local induction of Foxp3 cells than a commensal bacterium that only expresses one or two regulatory factors.

Both $\mathrm{mDCs}$ and $\mathrm{pDC}$ are present within the gastrointestinal tract in relatively high numbers compared with the peripheral blood. In the mouse, pDCs are numerically dominant within the lamina propria and Peyer's patches, while mDCs dominate in mesenteric lymph nodes. ${ }^{39}$ In murine models, pDCs have been shown to be essential for the induction and maintenance of oral tolerance as systemic depletion of pDCs prevents tolerance induction to fed antigen, while adoptive transfer of oral antigenloaded liver $\mathrm{pDCs}$ induced antigen-specific suppression of CD4 and CD8 Tcell responses. ${ }^{40}$ It is likely that $\mathrm{mDC}$ s and $\mathrm{pDCs}$ will also cooperate in the induction of tolerance to commensal microbes other than $B$ infantis, but our data suggest that not all Bifidobacteria induce the same $\mathrm{pDC}$ and $\mathrm{mDC}$ response.

$B$ infantis 35624 has been shown to protect against inflammatory disease in a number of murine models (including colitis, arthritis, respiratory allergy and infectious models). In humans, we now demonstrate in vivo that oral administration of this bacterium results in elevated IL-10 responses and Foxp3 expression in CD4 T cells and we describe the potential cellular mechanisms underpinning this regulatory response. Manipulation of $\mathrm{T}$ regulatory cell numbers or functions is an exciting therapeutic target in a wide range of inflammatory diseases. ${ }^{41} 42$ A clearer understanding of the mechanisms employed in vivo for the induction of oral tolerance by the microbiota will probably result in rational strategies to manipulate both regulatory and effector $\mathrm{T}$ cells, thereby influencing gastrointestinal disorders such as food allergy, eosinophilic oesophagitis, irritable bowel syndrome and inflammatory bowel diseases.

Funding The authors are supported by Swiss National Foundation grants (project numbers 32030-132899 and 310030-127356), Christine Kühne Center for Allergy Research and Education, Science Foundation Ireland and Alimentary Health Ltd.

Competing interests DG and BK are employees of the university campus company Alimentary Health Ltd. LOM, FS and EO are consultants to Alimentary Health Ltd. FS has received research grants from GSK. CA has received research support from Novartis and Stallergenes and consulted for Actellion, Aventis and Allergopharma. PK, $\mathrm{MZ}$, ReF and RuF have no conflict of interest. The content of this article was neither influenced nor constrained by these facts.

Ethics approval Clinical research ethics committee of the Cork Teaching Hospitals, Ireland.

Contributors PK, DG, MZ, RFrei, RFerstl and LOM performed the laboratory analysis and contributed to the interpretation of the data. FS, EQ, BK, CA and LOM contributed to study concept and design, analysis and interpretation of the data and drafting of the manuscript while LOM supervised the conduct of these studies.

Provenance and peer review Not commissioned; externally peer reviewed.

\section{REFERENCES}

1. O'Hara AM, Shanahan F. The gut flora as a forgotten organ. EMBO Rep 2006: 7:688-93.

2. Shanahan F. Gut microbes: from bugs to drugs. Am J Gastroenterol 2010:105:275-9.

3. Lee YK, Mazmanian SK. Has the microbiota played a critical role in the evolution of the adaptive immune system? Science 2010;330:1768-73.

4. Sakaguchi S, Miyara M, Costantino CM, et al. FOXP3 + regulatory T cells in the human immune system. Nat Rev Immunol 2010;10:490-500.

5. Akdis M, Verhagen J, Taylor A, et al. Immune responses in healthy and allergic individuals are characterized by a fine balance between allergen-specific $\mathrm{T}$ regulatory 1 and T helper 2 cells. J Exp Med 2004;199:1567-75.

6. O'Mahony C, Scully P, O'Mahony D, et al. Commensal-induced regulatory T cells mediate protection against pathogen-stimulated NF-kappaB activation. PLoS Pathog 2008; $4: \mathrm{e} 1000112$

7. Atarashi K, Tanoue T, Shima T, et al. Induction of colonic regulatory T cells by indigenous Clostridium species. Science 2011;331:337-41.

8. Karimi K, Inman MD, Bienenstock $J$, et al. Lactobacillus reuteri-induced regulatory $T$ cells protect against an allergic airway response in mice. Am J Respir Crit Care Med 2009;179:186-93

9. Di Giacinto C, Marinaro M, Sanchez M, et al. Probiotics ameliorate recurrent Th1 mediated murine colitis by inducing IL-10 and IL-10-dependent TGF-beta-bearing regulatory cells. J Immunol 2005; 174:3237-46.

10. Lyons A, O'Mahony D, O'Brien F, et al. Bacterial strain-specific induction of Foxp3+ T regulatory cells is protective in murine allergy models. Clin Exp Allergy 2010;40:811-19

11. Martínez-Cañavate A, Sierra S, Lara-Villoslada F, et al. A probiotic dairy product containing L. gasseri CECT5714 and L. coryniformis CECT5711 induces immunologica changes in children suffering from allergy. Pediatr Allergy Immunol 2009;20:592-600.

12. Pronio A, Montesani C, Butteroni C, et al. Probiotic administration in patients with ileal pouch-anal anastomosis for ulcerative colitis is associated with expansion of mucosal regulatory cells. Inflamm Bowel Dis 2008:14:662-8.

13. Atarashi K, Umesaki Y, Honda K. Microbiotal influence on T cell subset development. Semin Immunol 2011;23:146-53.

14. Coombes JL, Siddiqui KR, Arancibia-Cárcamo CV, et al. A functionally specialized population of mucosal CD103+ DCs induces Foxp3 + regulatory T cells via a TGFbeta and retinoic acid-dependent mechanism. J Exp Med 2007:204:1757-64.

15. Sun $\mathbf{C M}$, Hall JA, Blank RB, et al. Small intestine lamina propria dendritic cells promote de novo generation of Foxp3 T reg cells via retinoic acid. J Exp Med 2007:204:1775-85

16. Mazmanian SK, Liu CH, Tzianabos A0, et al. An immunomodulatory molecule of symbiotic bacteria directs maturation of the host immune system. Cell 2005:122:107-18.

17. Yamazaki S, Steinman RM. Dendritic cells as controllers of antigen-specific Foxp3+ regulatory T cells. J Dermatol Sci 2009;54:69-75.

18. Akdis M, Burgler S, Crameri R, et al. Interleukins, from 1 to 37 , and interferon- $\gamma$ : receptors, functions, and roles in diseases. J Allergy Clin Immunol 2011:127:701-21, e1-70.

19. Pulendran B, Tang $H$, Manicassamy S. Programming dendritic cells to induce $T(H) 2$ and tolerogenic responses. Nat Immunol 2010;11:647-55.

20. Manicassamy S, Ravindran R, Deng J, et al. Toll-like receptor 2-dependent induction of vitamin A-metabolizing enzymes in dendritic cells promotes $T$ regulatory responses and inhibits autoimmunity. Nat Med 2009;15:401-9.

21. Lai Y, Di Nardo A, Nakatsuji T, et al. Commensal bacteria regulate Toll-like receptor 3-dependent inflammation after skin injury. Nat Med 2009;15:1377-82. 
22. van der Aar AM, Sibiryak DS, Bakdash G, et al. Vitamin D3 targets epidermal and dermal dendritic cells for induction of distinct regulatory T cells. J Allergy Clin Immunol 2011;127:1532-40.e7.

23. Palomares $\mathbf{0}, 0^{\prime}$ Mahony $\mathrm{L}$, Akdis CA. The many routes of dendritic cells to ensure immune regulation. J Allergy Clin Immunol 2011;127:1541-2.

24. O'Mahony L, McCarthy J, Kelly P, et al. Lactobacillus and bifidobacterium in irritable bowel syndrome: symptom responses and relationship to cytokine profiles. Gastroenterology 2005;128:541-51.

25. O'Mahony L, O'Callaghan L, McCarthy J, et al. Differential cytokine response from dendritic cells to commensal and pathogenic bacteria in different lymphoid compartments in humans. Am J Physiol Gastrointest Liver Physiol 2006;290:G839-45.

26. Sibartie S, O'Hara AM, Ryan J, et al. Modulation of pathogen-induced CCL20 secretion from HT-29 human intestinal epithelial cells by commensal bacteria. BMC Immunol 2009;10:54.

27. Whorwell PJ, Altringer L, Morel J, et al. Efficacy of an encapsulated probiotic Bifidobacterium infantis 35624 in women with irritable bowel syndrome. Am J Gastroenterol 2006;101:1581-90.

28. O'Hara AM, O'Regan P, Fanning A, et al. Functional modulation of human intestinal epithelial cell responses by Bifidobacterium infantis and Lactobacillus salivarius. Immunology 2006:118:202-15.

29. van der Kleij H, O'Mahony C, Shanahan F, et al. Protective effects of Lactobacillus reuteri and Bifidobacterium infantis in murine models for colitis do not involve the vagus nerve. Am J Physiol Regul Integr Comp Physiol 2008;295:1131-7.

30. McCarthy J, O'Mahony L, O'Callaghan L, et al. Double blind, placebo controlled trial of two probiotic strains in interleukin 10 knockout mice and mechanistic link with cytokine balance. Gut 2003;52:975-80.
31. Miyara M, Sakaguchi S. Human FoxP3 $(+) \mathrm{CD} 4(+)$ regulatory T cells: their knowns and unknowns. Immunol Cell Biol 2011;89:346-51.

32. Hori S, Nomura T, Sakaguchi S. Control of regulatory T cell development by the transcription factor Foxp3. Science 2003;299:1057-61.

33. Duriancik DM, Lackey DE, Hoag KA. Vitamin A as a regulator of antigen presenting cells. J Nutr 2010;140:1395-9.

34. Maslowski KM, Mackay CR. Diet, gut microbiota and immune responses. Nat Immunol 2011;12:5-9.

35. Fukuda S, Toh $\mathrm{H}$, Hase $\mathrm{K}$, et al. Bifidobacteria can protect from enteropathogenic infection through production of acetate. Nature 2011;469:543-7.

36. Fukata M, Abreu MT. Pathogen recognition receptors, cancer and inflammation in the gut. Curr Opin Pharmacol 2009:9:680-7.

37. Round JL, Lee SM, Li J, et al. The Toll-like receptor 2 pathway establishes colonization by a commensal of the human microbiota. Science 2011:332:974-7.

38. Round JL, Mazmanian SK. Inducible Foxp3 + regulatory T-cell development by a commensal bacterium of the intestinal microbiota. Proc Natl Acad Sci U S A 2010;107:12204-9.

39. Wang $\mathbf{X}, 0^{\prime}$ Gorman MR, Bu HF, et al. Probiotic preparation VSL\#3 alters the distribution and phenotypes of dendritic cells within the intestinal mucosa in C57BL/ 10J mice. J Nutr 2009;139:1595-602.

40. Goubier A, Dubois B, Gheit $\mathrm{H}$, et al. Plasmacytoid dendritic cells mediate ora tolerance. Immunity 2008;29:464-75.

41. Akdis $\mathbf{M}$, Akdis CA. Therapeutic manipulation of immune tolerance in allergic disease. Nat Rev Drug Discov 2009;8:645-60.

42. O'Mahony L, Akdis M, Crameri R, et al. Novel immunotherapeutic approaches for allergy and asthma. Autoimmunity 2010;43:493-503.

Utilise our Quick Response code (QR) to sign up for our electronic table of contents (eTOC) alert.

To make this simple you can sign up now via your Smartphone.

\section{FOLLOWTHESE} THREE EASY STEPS:

1. Downbad a free $Q R$ reader from your handset's app store

2. Hold your Smartphone over the QR code

3. You will then be fonvarded to the eTOC sign up page

To find out more about $Q R$ codes visit

group.bmj.com/products/journals/qr-codes

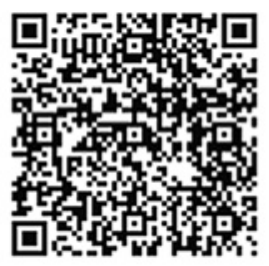

